\title{
Local noise analysis of a Schottky contact: Combined thermionic-emission-diffusion theory
}

\author{
G. Gomila, ${ }^{\text {a) }}$ O. M. Bulashenko, and J. M. Rubí \\ Departament de Física Fonamental, Universitat de Barcelona, Diagonal 647, E-08028 Barcelona, Spain
}

(Received 23 September 1997; accepted for publication 5 November 1997)

\begin{abstract}
A theoretical model for the noise properties of Schottky barrier diodes in the framework of the thermionic-emission-diffusion theory is presented. The theory incorporates both the noise induced by the diffusion of carriers through the semiconductor and the noise induced by the thermionic emission of carriers across the metal-semiconductor interface. Closed analytical formulas are derived for the junction resistance, series resistance, and contributions to the net noise localized in different space regions of the diode, all valid in the whole range of applied biases. An additional contribution to the voltage-noise spectral density is identified, whose origin may be traced back to the cross correlation between the voltage-noise sources associated with the junction resistance and those for the series resistance. It is argued that an inclusion of the cross-correlation term as a new element in the existing equivalent circuit models of Schottky diodes could explain the discrepancies between these models and experimental measurements or Monte Carlo simulations. (C) 1998 American Institute of Physics. [S0021-8979(98)01705-8]
\end{abstract}

\section{INTRODUCTION}

Schottky barrier diodes are being extensively used in different applications, such as mixers and detectors, due to their good high-frequency behavior. ${ }^{1}$ There is a vast literature devoted to the description of the transport properties, i.e., current-voltage $(\mathrm{I}-\mathrm{V})$ characteristics, capacitance, conductance, etc., of these devices. ${ }^{2-4}$ In recent years, however, special attention has been paid to the characterization of the noise properties of Schottky barrier diodes. ${ }^{5-8}$ The main approach currently used to interpret the experimental noisemeasurement results is the phenomenological equivalent noise circuit technique. ${ }^{5-7}$ Under this approach the diode is characterized by means of the corresponding equivalent circuit. Hence, by associating each resistive element of the circuit with different noise sources (shot noise for the junction resistance, thermal noise for the series resistance, etc.) the total noise of the device is evaluated. ${ }^{9}$ Despite its wide use in electrical engineering, it should be noted, however, that this phenomenological procedure does not offer a relation between the equivalent circuit elements and the kinetic and material parameters of a device (barrier height, mobility, doping, surface recombination velocity, etc.). Moreover, it implicitly assumes that only a given type of noise can be assigned to each equivalent-circuit element and that the different sources of noise are uncorrelated. While the first assumption seems to hold in most of the cases, the second assumption might not, due to the existence of spatial correlation between different active regions of the device. ${ }^{10}$

To overcome this situation, and to avoid any a priori assumption regarding the different sources of noise, more recently microscopic Monte Carlo (MC) simulation has been performed. ${ }^{8}$ Although the $\mathrm{MC}$ technique describes the carrier transport on the microscopic level and takes into account a

\footnotetext{
a)Electronic mail: gabriel@ffn.ub.es
}

large variety of kinetic processes, there are some difficulties in its application to the study of Schottky barrier diodes. In particular, when the barrier height is sufficiently large $\left(>5 k_{B} T / q\right)$ a depletion of carriers of several orders of magnitude occurs near the metal-semiconductor interface at low voltage biases. In such a case, the MC simulation becomes very time consuming because of poor statistics of carriers in the depletion layer, ${ }^{8}$ which, in turn, controls the current and noise properties under these conditions. To overcome the problem the special algorithm of particle multiplication has been realized to calculate the $\mathrm{I}-\mathrm{V}$ characteristics of the diode with a high barrier. ${ }^{11}$ Whether this algorithm is suitable for the noise calculation remains unclear. As a consequence, only the small-barrier diodes (near the flat-band conditions) have been simulated by the MC method. ${ }^{8}$ Therefore, the development of other methods able to provide theoretical analysis of the local noise properties of the Schottky contacts with arbitrary (low and high) barriers in the wide range of applied voltage biases seems to be highly desirable.

The purpose of the present article is to present precisely a simple analytical analysis of the different contributions to the net noise of a Schottky barrier diode. To this end we propose a combined model for the noise properties of these devices which takes into account in a common framework the two main sources of noise present in Schottky diodes, namely, the noise due to the thermionic emission of carriers across the metal-semiconductor interface and the noise due to the diffusion of carriers inside the semiconductor. By means of an analytical method recently developed ${ }^{10}$ we have obtained the explicit expressions for the local noise and impedance distributions, which allow us to identify the regimes of the system parameters, where one or another noise source dominates.

Furthermore, we show that the equivalent noise circuit previously reported for these contacts ${ }^{5-7}$ can be obtained as a limit of our model in some particular limiting cases. The 
equivalent-circuit parameters, such as the junction resistance, the series resistance, etc., acquire closed analytical formulas valid in the whole range of voltages including the crossover between the shot noise and thermal noise limits. In addition, our results suggest that in order to adequately describe the noise behavior of the Schottky diode under intermediate applied biases the traditionally used equivalent noise circuit model should be corrected by adding a new circuit element corresponding to the cross-correlation between the noise source associated with the junction resistance, and the noise source associated with the series resistance. This suggestion is based on the analytical expression for the cross-correlation term, and supported by our calculations.

The organization of the article is as follows: In Sec. II we review the thermionic-emission-diffusion theory which reflects the basic transport physics in an ideal $n$-type Schottky barrier diode. In Sec. III we propose the corresponding fluctuating thermionic-emission-diffusion model, which incorporates both the fluctuations due to the diffusion of carriers inside the semiconductor and the fluctuations induced by the thermionic emission of carriers across the metal-semiconductor interface. Then, the analytical solution of the model is derived, and the different contributions to the total noise and local noise distributions are identified. In Sec. IV the steady-state spatial profiles for the electric potential, electric field and carrier density are numerically obtained from thermionic-emission-diffusion equations, which will be used as input data to evaluate the noise characteristics. In Sec. V the equilibrium expressions for the analytical formulas found in Sec. III are derived and their compatibility with the Nyquist theorem shown. In Sec. VI the corresponding nonequilibrium results are presented and analyzed. Finally, in Sec. VII we sum up the main contributions of the article.

\section{THERMIONIC-EMISSION-DIFFUSION THEORY}

For ideal Schottky barrier diodes the thermionicemission-diffusion theory of Crowell and $\mathrm{Sze}^{12}$ has long been recognized as an appropriate theory to describe the transport properties of these devices. The theory assumes that the two main current-limiting processes occurring in a Schottky barrier diode are the diffusion and drift of carriers through the semiconductor part of the contact and the thermionic emission across the metal-semiconductor interface. These two processes are effectively in series, and the current is determined predominantly by the process which causes the larger impediment to the carrier flow or, in a general case, by a combined action of both of them if they are comparable. The latter was pointed out as the most realistic situation. ${ }^{3}$

The first process, electron transport through the semiconductor, is described by means of the drift-diffusion model, that is an equation for the current coupled self-consistently to the Poisson equation

$$
\begin{aligned}
& \frac{I(t)}{A}=q n v(E)+q D(E) \frac{\partial n}{\partial x}+\epsilon \frac{\partial E}{\partial t}, \\
& \frac{\partial E}{\partial x}=\frac{q}{\epsilon}\left(N_{D}-n\right),
\end{aligned}
$$

Here, $n(x, t)$ and $E(x, t)$ are the electron density and the electric field, respectively, and $A$ is the cross-sectional area. The drift velocity $v(E)$ and the diffusion coefficient $D(E)$ depend, in a general case, on the local electric field. In this model the current across any section of the device is the sum of the conducting current $J(x, t)$ (composed of the drift and diffusion components) and the displacement current. The current is conserved and equal to that in the external circuit $I(t)$.

The second current-limiting process, the thermionic emission, is described by means of a boundary condition for the conducting current at the top of the potential barrier, which is expressed in terms of an effective recombination velocity $v_{r}$ as ${ }^{12,13}$

$$
J(0, t)=q\left[n_{0}(t)-n_{0}^{\mathrm{eq}}\right] v_{r} A .
$$

The term with $n_{0} v_{r}$ represents the electron flux from semiconductor to metal, with $n_{0}$ being the electron density at the top of the barrier. The $n_{0}^{\mathrm{eq}} v_{r}$ represents the opposite flux from metal to semiconductor, where the quasiequilibrium electron density $n_{0}^{\mathrm{eq}}=N_{c} e^{-q \phi_{b} / k_{B} T}$ does not depend on the bias, $N_{c}$ is the semiconductor effective density of states, and $\phi_{b}$ the contact barrier height as seen from the metal side. The velocity $v_{r}$ is given by ${ }^{12} v_{r}=A^{*} T^{2} / q N_{c}$, where $A^{*}$ is the effective Richardson constant and $T$ is the lattice temperature.

In this article we are interested in modeling the lowfrequency plateau of the noise spectrum (above the $1 / f$-noise region), corresponding to the time scale much longer than the dielectric relaxation time. Due to the frequency range chosen, the displacement current can be neglected. Therefore, by eliminating $n$, the system (1), (2) yields a nonlinear second-order differential equation for the electric-field profile,

$$
D(E) \frac{d^{2} E}{d x^{2}}+v(E)\left(\frac{d E}{d x}-\frac{q}{\epsilon} N_{D}\right)=-\frac{I}{\epsilon A} .
$$

Moreover, by taking into account that in the frequency range of interest $J(0, t)=I$, the boundary condition at $x=0$ for Eq. (4) following from Eqs. (3) and (2) becomes

$$
\left.\frac{d E}{d x}\right|_{x=0}=\frac{q}{\epsilon}\left[N_{D}-n_{0}^{\mathrm{eq}}-\frac{I}{q v_{r} A}\right] .
$$

On the opposite side of the semiconductor the flat-band boundary condition is assumed, that is

$$
\left.\frac{d E}{d x}\right|_{x=L}=0,
$$

where the sample length $L$ is chosen to be large enough, i.e., much larger than the Debye screening length $L_{D}=\left(\epsilon k_{B} T / q^{2} N_{D}\right)^{1 / 2}$ to guarantee the quasineutrality condition $n(L)=N_{D}$. (This condition is also equivalent to putting the Ohmic contact at $x=L$.) The results for a short Schottky barrier diode ( $L$ of the order of $L_{D}$ ) will be published elsewhere. ${ }^{14}$

To distinguish different regimes in the thermionicemission-diffusion theory, it is useful to introduce some dimensionless parameters as follows: Since the width of the 
depletion layer forming the potential barrier is of the order of several Debye lengths $L_{D}$, the typical absolute value of the maximum of the electric field at the top of the barrier can be measured in units of $E_{\mathrm{th}}=k_{B} T /\left(q L_{D}\right)$. Accordingly, the characteristic diffusion velocity $v_{D}$ appeared in the theory ${ }^{12}$ is of the order of $\mu E_{\mathrm{th}}$, with $\mu=\left.v^{\prime}(E)\right|_{E=0}$ being the lowfield mobility. Therefore, the parameter $\beta=\mu E_{\mathrm{th}} / v_{r}$ can be used to estimate the importance of different regimes limiting the current. We will see in Sec. VI when we compute the impedance of the diode, that for $\beta \ll 1$ the transport is diffusion limited while for $\beta \gg 1$ it is thermionic-emission limited (for $\beta \sim 1$ both processes contribute similarly).

Notice, that the parameter $\beta$ only enters into the boundary condition (5), where the term with the current is proportional to $\beta$. Therefore, in the diffusive limit when $\beta \rightarrow 0$, this term is small in respect to others, and the electron density $n_{0}$ remains approximately at the equilibrium value $n_{0}^{\mathrm{eq}}$ and is not altered by the applied voltage (current). This is equivalent to assuming that at the interface the quasi-Fermi level in the semiconductor coincides with the Fermi level in the metal. ${ }^{2}$ In this case the decrease of the quasi-Fermi level occurs through the depletion region. In the opposite thermionicemission limit when $\beta$ is large and the boundary condition at the interface depends strongly on the current, the quasiFermi level for electrons remains flat throughout the depletion region (the mobility is large) and drops down inside the metal. Therefore, the boundary condition (5) mirrors the behavior of the quasi-Fermi level nearby the metalsemiconductor contact.

\section{FLUCTUATIONS IN SCHOTTKY BARRIER DIODES}

\section{A. General formulation}

According to the thermionic-emission-diffusion theory described in the previous section two current limiting processes are assumed to exist in Schottky barrier diodes: the diffusion of carriers through the semiconductor and the thermionic emission of carriers across the metal-semiconductor interface. Consequently, by associating, as usual, a noise source with each current-limiting process, two different sources of noise should be present in the system: a diffusion noise source and a thermionic-emission one. ${ }^{15}$ While the former accounts for the different random scattering processes occurring inside the semiconductor, the latter describes the random nature of the thermionic-emission processes across the metal-semiconductor interface. Although the existence of both noise sources seems to be out of discussion, none of the existing carrier-transport models incorporates them in a unified way. Concerning the equivalent circuit model, it does not incorporate them in a proper way, as will be shown below.

Here we present a simple model in which both sources of noise are taken into account in a common framework. The diffusion noise is modeled, as usual, as a bulk current noise source which accounts for the velocity fluctuations ${ }^{16}$

$$
K(x)=4 q^{2} n(x) D(x),
$$

where the nonlocal heating of carriers is neglected.
On the other hand, we model the source of noise due to the thermionic emission of carriers across the metalsemiconductor interface as a surface noise source associated with a stochastic boundary condition. Its spectral density, as shown in Appendix A, is given by

$$
S_{I \mathrm{c}}=2 q\left[I+2 I_{c}\right] \text {, }
$$

with $I_{c}=q n_{0}^{\mathrm{eq}} v_{r} A$. This approximation is based on the assumption that the thermionic-emission processes occur inside a layer of the order of the electron mean free path $\lambda_{p}$ which is assumed to be a small fraction of the barrier width. The two sources of noise, as well as two local noise sources of Eq. (7) corresponding to different slices $x$ and $x^{\prime}$ are assumed to be uncorrelated, since the characteristic scale is such that $\left|x-x^{\prime}\right| \gg \lambda_{p}$.

As pointed out by Van Vliet, ${ }^{17,18}$ in the case that surface as well as bulk noise sources are present in a given system the most convenient method to analyze the fluctuations and noise properties of the system is the Langevin response formulation. Essentially, this formulation consists in adding to carrier transport equations the corresponding stochastic noise sources and then, by solving these equations and computing the appropriate averages, to obtain the statistical properties of the different magnitudes of interest. For the case under consideration, this means that the fluctuation of the electric field $\delta E_{x}$ at slice $x$ should satisfy the linearized version of Eq. (4) with a source noise term for the current $\delta I_{x}$

$$
\epsilon A \hat{L} \delta E_{x}=-\delta I_{x},
$$

with the operator $\hat{L}$ given by

$$
\begin{aligned}
\hat{L}= & D(E) \frac{d^{2}}{d x^{2}}+v(E) \frac{d}{d x}+D^{\prime}(E) \frac{d^{2} E}{d x^{2}} \\
& +v^{\prime}(E)\left(\frac{d E}{d x}-\frac{q}{\epsilon} N_{D}\right) .
\end{aligned}
$$

Here, $\delta I_{x}$ represents the stochastic current induced by the random scattering of carriers inside the semiconductor. It has zero mean and $\delta$-type correlation function $\left\langle\delta I_{x} \delta I_{x^{\prime}}\right\rangle=A K(x) \Delta f \delta\left(x-x^{\prime}\right)$, with $K(x)$ defined through Eq. (7) and $\Delta f$ the frequency bandwidth. Furthermore, at the metal-semiconductor interface the fluctuations satisfy the linearized version of Eq. (5) with a surface Langevin-like term $\delta I_{s}$ present. Explicitly,

$$
\left.\frac{d \delta E_{x}}{d x}\right|_{x=0}=-\frac{\delta I_{s}}{\epsilon v_{r} A} .
$$

Here, $\delta I_{s}$ describes the random nature of the thermionic emission of carriers across the metal-semiconductor interface. It has zero mean and the dispersion $\left\langle\delta I_{s}^{2}\right\rangle=S_{I \mathrm{c}} \Delta f$ with $S_{\text {Ic }}$ given by Eq. (8).

Due to the requirement on the length of the sample $L \gg L_{D}$ and the flat boundary condition at $x=L$, for the fluctuations of the field at this boundary we have

$$
\begin{aligned}
& \delta E_{L}=0, \\
& \left.\frac{d \delta E_{x}}{d x}\right|_{x=L}=0,
\end{aligned}
$$


with the meaning that the contribution to the noise at the contact from distances much larger than the Debye length is screened out.

Equations (7)-(12) constitute a complete set of equations to analyze the noise properties of Schottky barrier diodes in the thermionic-emission-diffusion approximation.

\section{B. Analytical solution}

Note that $\hat{L}$ is a linear second-order differential operator with space-dependent coefficients. To find the solution in an analytical form we use the method recently applied for the $n^{+} n$ semiconductor junction. ${ }^{10}$ The general solution for the electric-field fluctuation is given by

$$
\begin{aligned}
\delta E_{x}= & C_{1} \rho(x)+C_{2} u(x)+\rho(x) \int_{0}^{x} \frac{u(\xi)}{\epsilon A D(\xi) W(\xi)} \delta I_{\xi} d \xi \\
& +u(x) \int_{x}^{L} \frac{\rho(\xi)}{\epsilon A D(\xi) W(\xi)} \delta I_{\xi} d \xi,
\end{aligned}
$$

where $W(x)=\rho(x) u^{\prime}(x)-u(x) \rho^{\prime}(x)$ is the Wronskian, and $\rho(x)$ and $u(x)$ are auxiliary functions, satisfying the equations $\hat{L} \rho(x)=0, \hat{L} u(x)=0$, i.e., they are the solutions of the homogeneous equation corresponding to Eq. (9). The expressions for the auxiliary functions are as follows: ${ }^{10}$

$$
\begin{aligned}
& \rho(x)=d E / d x, \\
& u(x)=\rho(x) \int_{C}^{x} \frac{W(\xi)}{\rho^{2}(\xi)} d \xi, \\
& W(x)=W(0) \exp \left\{-\int_{0}^{x} v\left[E\left(x^{\prime}\right)\right] / D\left[E\left(x^{\prime}\right)\right] d x^{\prime}\right\} .
\end{aligned}
$$

The value of the integration constant $W(0)$ is not actually important, since it will be canceled after substituting Eq. (14b) into (13), so we can assume $W(0)=1$. The constant $C$ in Eq. (14b) can be defined by the appropriate choice for the boundary condition of the function $u(x)$. Since it does not influence on the final results, we take $u^{\prime}(0)=0$ which corresponds to the homogeneous boundary conditions for the Green functions of the operator $\hat{L}$ and provides the most compact intermediate expressions. The integration constants $C_{1}$ and $C_{2}$ in Eq. (13) are determined by the boundary conditions at $x=0$ and $x=L$. The condition (12) implies $\rho(L)=0$ and, hence, $C_{2}=0$. By differentiating Eq. (13) and using Eq. (11) we obtain the stochastic value for the constant $C_{1}$ as

$$
C_{1}=-\frac{\delta I_{s}}{\epsilon v_{r} A \rho_{0}^{\prime}}
$$

Hereafter, the prime stands for a derivative, and the subindex 0 refers to the value of the corresponding function at $x=0$.

The final expression for $\delta E_{x}$ then reads,

$$
\begin{aligned}
\delta E_{x}= & -\frac{\rho(x)}{\epsilon v_{r} A \rho_{0}^{\prime}} \delta I_{s}+\rho(x) \int_{0}^{x} \frac{u(\xi)}{\psi(\xi)} \delta I_{\xi} d \xi \\
& +u(x) \int_{x}^{L} \frac{\rho(\xi)}{\psi(\xi)} \delta I_{\xi} d \xi,
\end{aligned}
$$

where we have denoted $\psi(\xi)=\epsilon A D(\xi) W(\xi)$. Equation (16), together with Eq. (14), constitutes the analytical solution to the problem posed by Eqs. (9)-(12). It expresses the electric-field fluctuations at point $x$ in terms of the noise sources $\delta I_{x}, \delta I_{s}$ and the stationary electric field profile which appears through $\rho(x), u(x)$, and $\psi(x)$.

It is worth noting that Eq. (16), apart from allowing us to derive explicit expressions for the impedance field and voltage and current fluctuations (see below), it could also be used to evaluate the spatial correlations of the electric-field fluctuations $\left\langle\delta E_{x} \delta E_{x^{\prime}}\right\rangle$ between two different points (regions) under nonhomogeneous conditions. These correlations are active over several characteristic screening lengths of the system. ${ }^{10}$

Now, we are in a position to evaluate the fluctuation in the voltage at the terminal ends by integrating the fluctuation of the electric field given through Eq. (16) along the system,

$$
\delta V=\int_{0}^{L} \delta E_{x} d x
$$

We then obtain,

$$
\delta V=Z_{c} \delta I_{s}+\int_{0}^{L} \nabla Z(x) \delta I_{x} d x,
$$

where

$$
Z_{c}=\frac{E_{0}-E_{L}}{\epsilon v_{r} A \rho_{0}^{\prime}}
$$

is the impedance of the metal-semiconductor contact associated with the thermionic-emission processes, and $\nabla Z(x)$ the bulk impedance field associated with the drift-diffusion processes in the semiconductor. The latter consists of two terms

$$
\begin{aligned}
& \nabla Z(x)=\nabla Z_{\text {dep }}(x)+\nabla Z_{\text {ser }}(x), \\
& \nabla Z_{\text {dep }}(x)=\frac{\rho(x)}{\psi(x)} \frac{E_{0}-E_{L}}{\rho_{0} \rho_{0}^{\prime}}, \\
& \nabla Z_{\text {ser }}(x)=\frac{\rho(x)}{\psi(x)} \int_{0}^{x}\left[E_{L}-E\left(x^{\prime}\right)\right] \frac{W\left(x^{\prime}\right)}{\rho^{2}\left(x^{\prime}\right)} d x^{\prime} .
\end{aligned}
$$

It will be shown below, that the term $\nabla Z_{\text {dep }}$ is the contribution coming from the depletion layer and it dominates at low currents, while $\nabla Z_{\text {ser }}$ is determined by the doping level inside the semiconductor and is associated with the series resistance. The latter dominates at high currents when the depletion layer disappears (flat-band condition) and the voltage drops mainly on the bulk of the semiconductor.

From Eq. (18) the total impedance $Z$ of the Schottky contact, as well as the spectral density of the voltage fluctuations $S_{V}$, may be easily computed. One obtains, 


$$
\begin{aligned}
Z & =Z_{c}+\int_{0}^{L} \nabla Z_{\mathrm{dep}}(x) d x+\int_{0}^{L} \nabla Z_{\mathrm{ser}}(x) d x \\
& \equiv Z_{c}+Z_{\mathrm{dep}}+Z_{\mathrm{ser}}, \\
S_{V} & =Z_{c}^{2} S_{I \mathrm{c}}+A \int_{0}^{L}\left[\nabla Z_{\mathrm{dep}}(x)+\nabla Z_{\mathrm{ser}}(x)\right]^{2} K(x) d x \\
& \equiv S_{V \mathrm{c}}+S_{V \text { dep }}+S_{V \text { ser }}+S_{V \text { cros }},
\end{aligned}
$$

where

$$
\begin{aligned}
& S_{V \mathrm{c}}=Z_{c}^{2} S_{I \mathrm{c}}, \\
& S_{V \text { dep }}=A \int_{0}^{L}\left[\nabla Z_{\mathrm{dep}}(x)\right]^{2} K(x) d x, \\
& S_{V \text { ser }}=A \int_{0}^{L}\left[\nabla Z_{\mathrm{ser}}(x)\right]^{2} K(x) d x, \\
& S_{V \text { cros }}=A \int_{0}^{L} 2\left[\nabla Z_{\text {dep }}(x)\right]\left[\nabla Z_{\text {ser }}(x)\right] K(x) d x,
\end{aligned}
$$

and they represent different contributions to the total voltage fluctuations. On the other hand, we shall use the function $s_{V}(x)=[\nabla Z(x)]^{2} K(x)$, also subdivided into the corresponding components $s_{V \text { dep }}(x)+s_{V \text { cros }}(x)+s_{V \text { ser }}(x)$, in order to characterize the local contribution of different space regions to the net noise measured between the terminals.

The spectral density of the current fluctuations $S_{I}$ can be also computed by using Eqs. (23) and (24) as

$$
S_{I}=\frac{S_{V \mathrm{c}}+S_{V \mathrm{dep}}+S_{V \text { cros }}+S_{V \mathrm{ser}}}{\left(Z_{c}+Z_{\mathrm{dep}}+Z_{\mathrm{ser}}\right)^{2}} .
$$

Finally, the noise temperature $4 k_{B} T_{n}=S_{V} / Z$ takes on the form

$$
T_{n}=\frac{1}{4 k_{B}} \frac{S_{V \mathrm{c}}+S_{V \mathrm{dep}}+S_{V \mathrm{cros}}+S_{V \mathrm{ser}}}{Z_{c}+Z_{\mathrm{dep}}+Z_{\mathrm{ser}}} .
$$

Equations (23)-(30), once Eqs. (19)-(22) are used, give the exact expressions for the total diode impedance, voltage and current fluctuations, and the noise temperature as obtained form the thermionic-emission-diffusion theory. Furthermore, they also give information about the spatial distribution of the impedance throughout the device by means of the impedance field $\nabla Z(x)$, and the local contributions to the voltage fluctuations by virtue of the spatial profile $s_{V}(x)$. These local distributions may be very useful in interpreting the results as will be seen in what follows. It is worth noting that in order to compute Eqs. (19)-(22), and hence Eqs. (23) -(30), we only need to know the stationary electric field profile.

Recalling now the equivalent-circuit-noise formula for the current spectral density of the Schottky diode ${ }^{5}$

$$
S_{I}=\frac{2 q I R_{j}^{2}}{\left(R_{s}+R_{j}\right)^{2}}+\frac{4 k_{B} T R_{s}}{\left(R_{s}+R_{j}\right)^{2}},
$$

with $R_{s}$ and $R_{j}$ the series and the junction resistances, respectively, and comparing it with our Eq. (29), it is seen, that we arrived at a more general result. In particular, the junction resistance $R_{j}$ usually used as a phenomenological parameter to fit the experimental $I-V$ or noise characteristics, now can be evaluated from $R_{j}=Z_{c}+Z_{\text {dep }}$ and is given analytically by Eqs. (19) and (21). The series resistance $R_{s}$ is equal to $Z_{\text {ser }}$ in our model and can be obtained from Eq. (22). The noise associated with the junction resistance $2 q I R_{j}^{2}$ is given in our model through $S_{V \mathrm{c}}+S_{V \text { dep }}$, which are followed from Eqs. (25) and (26). Moreover, the noise term associated with the series resistance $4 k_{B} T R_{s}$ can be evaluated from $S_{V \text { ser }}$ given by Eq. (27). Finally, an additional term represented by $S_{V \text { cros }}$ absent in the equivalent circuit model has appeared in our theory, which is relevant, as will be shown below, in a certain range of voltages. It is interpreted as a cross-correlation between the voltage-noise sources associated with the junction resistance and those for the series resistance. More detailed discussion of all the terms will be found in the subsequent sections.

\section{STEADY-STATE SPATIAL PROFILES AND $I-V$ PLOTS}

In order to evaluate the different expressions found in the previous section, we need to compute the corresponding stationary profiles for the electric field. To find these profiles we solve numerically Eq. (4) with the boundary conditions (5) and (6) by making use of a finite difference scheme. For the sake of completeness, also the electron density and potential profiles will be reported. It can be shown that the diode behavior is governed by three dimensionless parameters [for given shapes of $v(E)$ and $D(E)$ ]: (i) the coefficient $\beta=\mu E_{\mathrm{th}} / v_{r}$ introduced in Sec. II characterizing the type of transport regime; (ii) the ratio $\alpha=n_{0}^{\mathrm{eq}} / N_{D}$ which characterizes the height of the contact barrier, and (iii) the current, for which we use the following units $J=I / I_{R}$, with $I_{R}=q \mu E_{\mathrm{th}} N_{D} A$.

In the following we shall use three different values of the parameter $\beta$ for a comparative analysis of different transport regimes: $\beta=20$ for the case of thermionic-emission (TE) dominated transport, $\beta=0.01$ for the case of diffusion (D) dominated transport, and $\beta=1$ for the intermediate thermionic-emission-diffusion (TE-D) regime.

The parameter $\alpha$ describes basically the nature of the contact under consideration. In this sense, for a rectifying contact (high barrier) $\alpha$ is very small, while for not rectifying contacts (low barrier) $\alpha \gtrsim 1$. In the following for our calculations we take $\alpha=10^{-7}$. This value provides a sufficiently high potential barrier $\left(\approx 16 k_{B} T / q\right.$ at equilibrium $)$ and a "deep" depletion layer with the electron concentration at the barrier top $n_{0} \sim 10^{-7} N_{D}$. For simplicity we consider only the case of constant mobility $\mu$ and the diffusion coefficient $D$, although explicit field dependencies characteristic for the hot carrier transport may be used without additional difficulties. To be concrete, we take $v(E)=\mu E, D=\mu k_{B} T / q$. The length of the sample is taken to be $L=15 L_{D}$ to guarantee the quasineutrality conditions at $x=L$.

Having found the electric-field profiles $E(x)$ for a given current $J$, the distributions of the potential $\phi(x)$ and the electron density $n(x)$ can be easily restored. Knowing the total voltage drop $V=\int_{0}^{L} E(x) d x+V_{\mathrm{bi}}$, with the equilibrium 


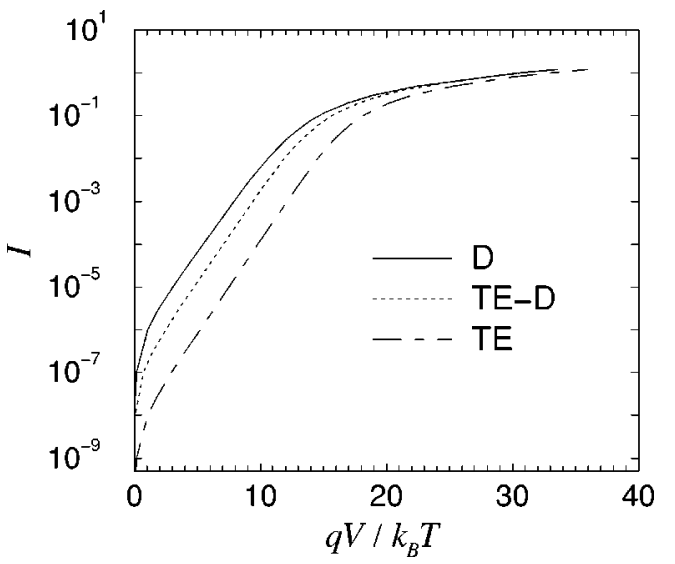

FIG. 1. $I-V$ characteristics for a Schottky diode operating at different regimes: D, TE-D, TE. Current is in units of $I_{R}=q \mu E_{\mathrm{th}} N_{D} A$.

built-in voltage $V_{\mathrm{bi}}=\left(k_{B} T / q\right) \ln (1 / \alpha)$, the $I-V$ curves can then be obtained. In our calculations in which $\alpha=10^{-7}$, the built-in voltage $V_{\mathrm{bi}} \approx 16 k_{B} T / q$.

A comparison of the $I-V$ characteristics for the structures simulated for different values of $\beta$ is shown in Fig. 1. In all cases the qualitative behavior is similar: for low biases, when the current is controlled by the depletion layer, there appears an exponential region, while for high biases the characteristics becomes linear displaying the presence of series resistance effects once the depletion layer has disappeared. Apart from a quantitative difference, the exponential behavior is seen to be more pronounced for the case TE. The fact that both the thermionic emission processes as well as the diffusion processes (or a combined action of both) may induce a similar behavior on the $I-V$ characteristics has to be taken into account in analyzing the properties of these diodes. In particular, care should be taken in drawing conclusion from an analysis of the $I-V$ characteristics in respect to the dominant transport mechanisms acting on a given system. In general, precise information on the values of the different contact parameters, i.e., recombination velocity, mobility, doping, etc., are necessary to arrive at a correct interpretation of the results. As we will see, the same statement holds for the analysis of the noise properties of these systems.

The steady-state spatial profiles for the case D and for different currents $J$ are shown in Fig. 2. The profiles display the usual behavior: for low currents a pronounced depletion layer is observed near the metal-semiconductor interface, while at higher currents the depletion layer progressively disappears [Fig. 2(a)]. Along with a disappearance of the depletion layer, the potential barrier also disappears [Fig. 2(c)]. The spatial distributions for another limiting case, TE case, are presented in Fig. 3. Apart from quantitative differences arisen from different typical scale of the current, it is seen that for the TE case at high enough currents the depletion layer can be substituted by an accumulation layer.

\section{EQUILIBRIUM NOISE AND THE NYQUIST THEOREM}

The relative significance of the different terms contributing to the impedance and noise of the Schottky diode can

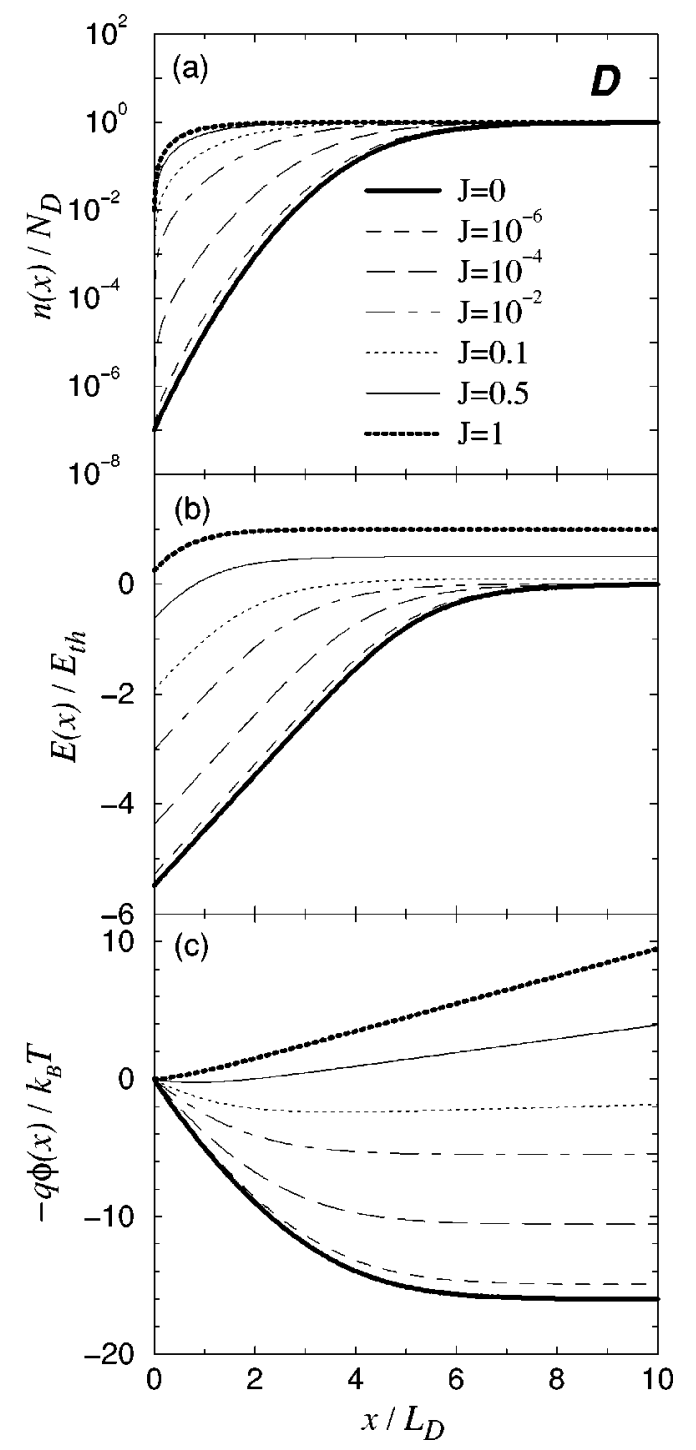

FIG. 2. Stationary distributions of the electron density $n$ (a), the electric field $E(\mathrm{~b})$, and the electric potential $\phi(\mathrm{c})$ of a Schottky diode in the regime D for different currents $J$.

be ascertained by considering the equilibrium case $I=0$, for which very transparent expressions can be obtained. For this case the equation for the balance of the current $n E+\left(k_{B} T / q\right) d n / d x=0$ can be integrated, giving

$$
n(x)=n_{0}^{\mathrm{eq}} \exp \left[q \phi(x) / k_{B} T\right],
$$

where we have used the boundary condition for the electric potential $\phi(0)=0$.

Furthermore, all the expressions are simplified, giving $\quad E(L)=0 ; \quad W(x)=\exp \left[q \phi(x) / k_{B} T\right] ; \quad E(x) W(x)$ $=L_{D 0}^{2}(d \rho / d x)$, where $L_{D 0}=\left(\epsilon k_{B} T / q^{2} n_{0}^{\mathrm{eq}}\right)^{1 / 2}$ is the screening length corresponding to the electron density at the interface. Substituting these relations into Eqs. (20)-(22) we obtain

$$
\begin{aligned}
& \nabla Z_{\mathrm{dep}}^{\mathrm{eq}}(x)=R(x) \frac{\rho(x)}{\rho(0)}, \\
& \nabla Z_{\mathrm{ser}}^{\mathrm{eq}}(x)=R(x)\left[1-\frac{\rho(x)}{\rho(0)}\right],
\end{aligned}
$$




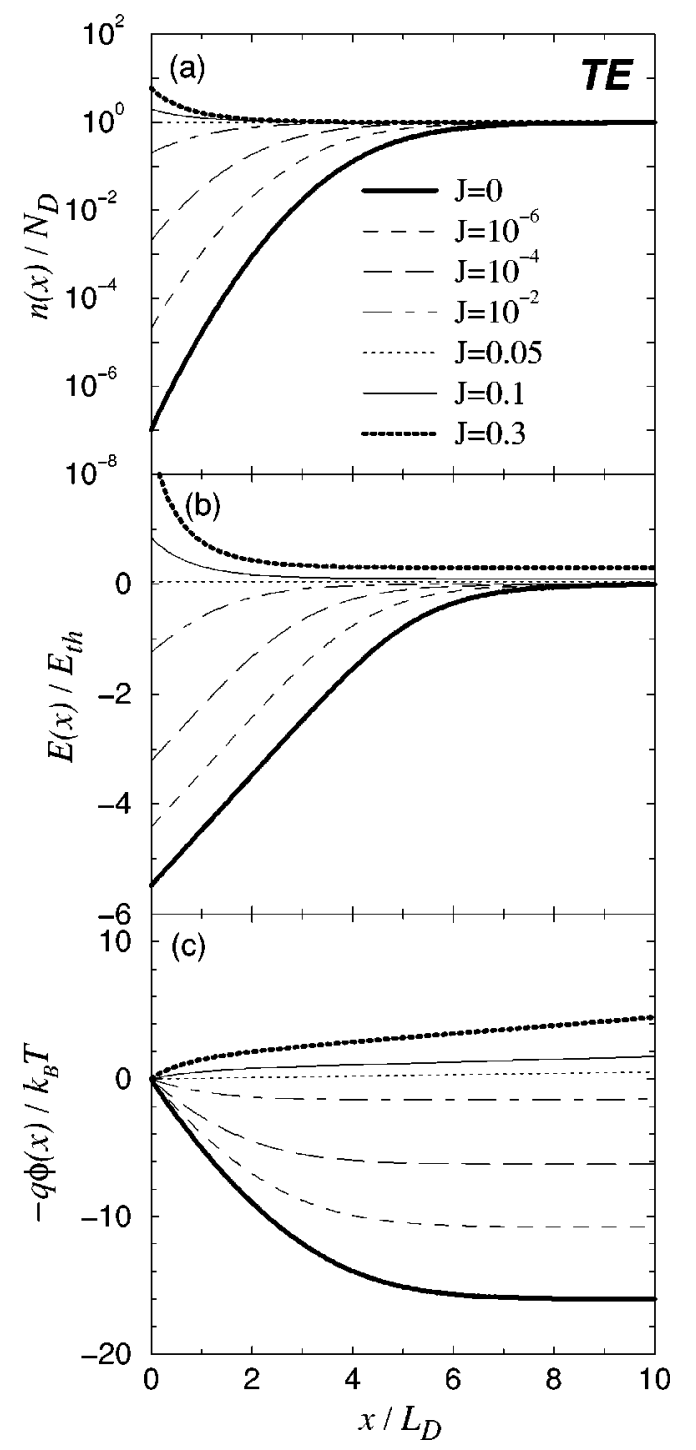

FIG. 3. Stationary distributions of the electron density $n$ (a), the electric field $E(\mathrm{~b})$, and the electric potential $\phi$ (c) of a Schottky diode in the regime TE for different currents $J$.

$$
\nabla Z^{\mathrm{eq}}(x)=R(x) \equiv \frac{1}{q \mu A n(x)},
$$

showing that the bulk impedance field is equal to the local (per unit length) resistance.

Figure 4 shows the spatial profiles for $\nabla Z^{\mathrm{eq}}(x)$ and its constituents $\nabla Z_{\text {dep }}^{\mathrm{eq}}(x)$ and $\nabla Z_{\text {ser }}^{\mathrm{eq}}(x)$ calculated by using the equilibrium distribution $n(x)$ shown in Figs. 2 or 3 (at equilibrium they are identical for all three transport regimes). The term $\nabla Z_{\mathrm{dep}}^{\mathrm{eq}}(x)$ is seen to give the main contribution near the interface $x<5 L_{D}$, since it represents the depletion layer contribution, in which the carrier density is very small. Whereas for $x>7 L_{D}$ the impedance is determined mainly by the series resistance term $\nabla Z_{\text {ser }}^{\text {eq }}$ which is constant over almost the whole structure and is determined by the donor density.

The contact impedance given by Eq. (19) is estimated by taking into account the relation $\rho_{0}^{\prime}=E_{0} / L_{D 0}^{2}$ valid at equilibrium. One gets

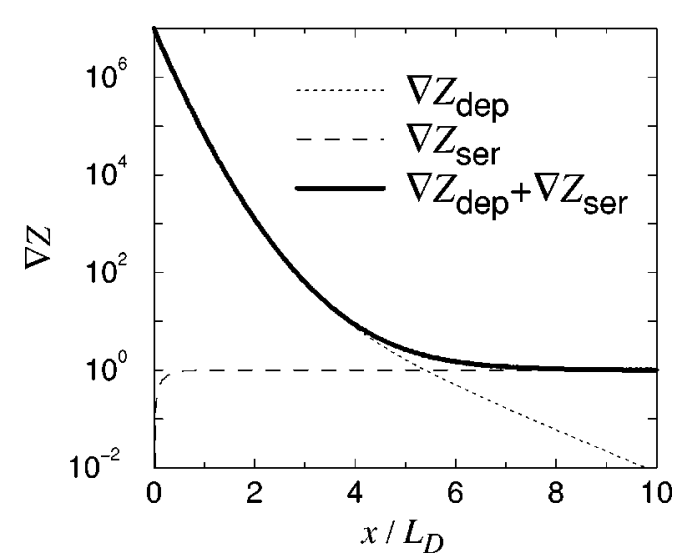

FIG. 4. Equilibrium bulk impedance field $\nabla Z^{\mathrm{eq}}(x)$ and its components $\nabla Z_{\text {dep }}^{\mathrm{eq}}(x)$ and $\nabla Z_{\text {ser }}^{\mathrm{eq}}(x)$, all normalized by $\left(q \mu A N_{D}\right)^{-1}$.

$$
Z_{c}=\frac{k_{B} T}{q^{2} n_{0}^{\mathrm{eq}} v_{r} A}=\frac{k_{B} T}{q I_{c}} \equiv R_{c},
$$

where $R_{c}$ is the well-known contact resistance, ${ }^{4}$ used to characterize the nature of the contact. Thus, the total equilibrium impedance (resistance) of the diode is given by $Z^{\mathrm{eq}}=R_{c}+\int_{0}^{L} R(x) d x=R_{c}+R_{\mathrm{dep}}+R_{\mathrm{ser}}$.

After integration along the structure the depletion-layer resistance is much greater than the series resistance, because $R_{\mathrm{dep}} / R_{\mathrm{ser}} \sim 1 / \alpha \gg 1$, as it should be for a rectifying contact. The total equilibrium diode resistance is then governed by the sum $R_{c}+R_{\mathrm{dep}}$. Introducing the characteristic series resistance as $R_{R}=E_{t h} L_{D} / I_{R}=L_{D} /\left(q \mu N_{D} A\right)$ the contact resistance can be evaluated by $R_{c}=R_{R}(\beta / \alpha)$, whereas the depletion resistance $R_{\mathrm{dep}} \approx R_{R} / \alpha$. Therefore, $R_{c} / R_{\mathrm{dep}} \approx \beta$, and one or another resistance dominates $\left(R_{c}\right.$ or $\left.R_{\text {dep }}\right)$ depending on the regime considered (TE or D).

A similar spatial analysis is performed for the voltage fluctuations. For the local noise contributions in Eqs. (26)(28) one gets

$$
\begin{aligned}
& s_{V \text { dep }}^{\mathrm{eq}}(x)=4 k_{B} T\left[\frac{\rho(x)}{\rho(0)}\right]^{2} R(x), \\
& s_{V \text { ser }}^{\mathrm{eq}}(x)=4 k_{B} T\left[1-\frac{\rho(x)}{\rho(0)}\right]^{2} R(x), \\
& s_{V \text { cros }}^{\mathrm{eq}}(x)=8 k_{B} T\left[1-\frac{\rho(x)}{\rho(0)}\right] \frac{\rho(x)}{\rho(0)} R(x) .
\end{aligned}
$$

Hence, for the net local noise $s_{V}^{\mathrm{eq}}(x)=s_{V \text { dep }}^{\mathrm{eq}}(x)$ $+s_{V \text { cros }}^{\mathrm{eq}}(x)+s_{V \text { ser }}^{\mathrm{eq}}(x)=4 k_{B} T R(x)$, which is the Nyquist theorem, since $R(x)$ is the local resistance. It should be pointed out that in recovering the Nyquist theorem all contributions, including the cross-correlation term, have been necessary.

The local noise distributions Eqs. (37)-(39) calculated by using the equilibrium profile $n(x)$ are shown in Fig. 5. As a consequence of the Nyquist theorem the thick solid lines in Figs. 4 and 5 coincide. Note that near the interface the main contribution to $s_{V}^{\mathrm{eq}}(x)$ comes from the depletion-layer term $s_{V \text { dep }}^{\text {eq }}(x)$. The series-noise term dominates in the quasineutral 


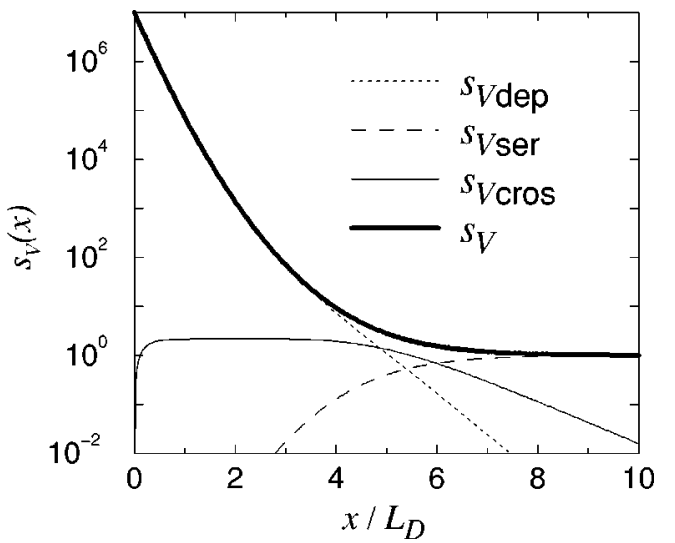

FIG. 5. Spatial profiles for the contributions $s_{V \text { dep }}^{\mathrm{eq}}(x), s_{V \text { cros }}^{\mathrm{eq}}(x)$, and $s_{V \text { ser }}^{\mathrm{eq}}(x)$ to the net local equilibrium noise $s_{V}^{\mathrm{eq}}(x)$, all normalized by $4 k_{B} T \Delta f /\left(\mu A N_{D}\right)$.

region, and the cross-correlation term is active in the crossover from the depletion layer to the quasineutral part of the diode.

For the contact contribution to the voltage noise we get by means of Eqs. (8), (25), and (36),

$$
S_{V \mathrm{c}}^{\mathrm{eq}}=4 q I_{c} Z_{c}^{2}=4 k_{B} T R_{c}
$$

which again consists of the Nyquist theorem. The total voltage fluctuations are then given by $S_{V}=4 k_{B} T\left(R_{\text {ser }}+R_{\text {dep }}+R_{c}\right)$. Similarly, we obtain for the current fluctuations $S_{I}=4 k_{B} T /\left(R_{\text {ser }}+R_{\text {dep }}+R_{c}\right)$. These results show that the Nyquist theorem is satisfied both locally and globally, as should be.

\section{NONEQUILIBRIUM FLUCTUATIONS IN A SCHOTTKY DIODE}

In this section we will discuss the results obtained from our theory for the nonequilibrium case. Note that to evaluate the different expressions derived in Sec. III B we only need to use the stationary electric-field profiles obtained in Sec. IV.

In Fig. 6 we have plotted the bulk impedance field $\nabla Z(x)$ and its components $\nabla Z_{\text {dep }}(x), \nabla Z_{\text {ser }}(x)$ for different

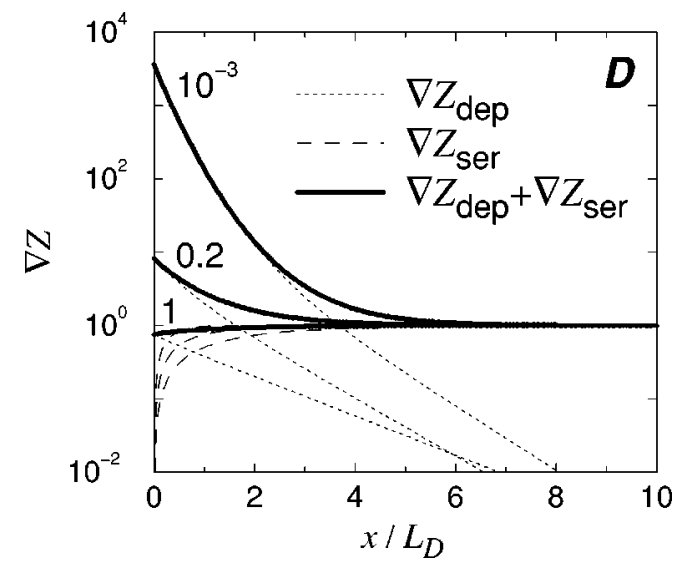

FIG. 6. Spatial profiles for the local impedance $\nabla Z(x)$ [normalized by $\left(q \mu A N_{D}\right)^{-1}$ ] for different currents $J$ for the case D.

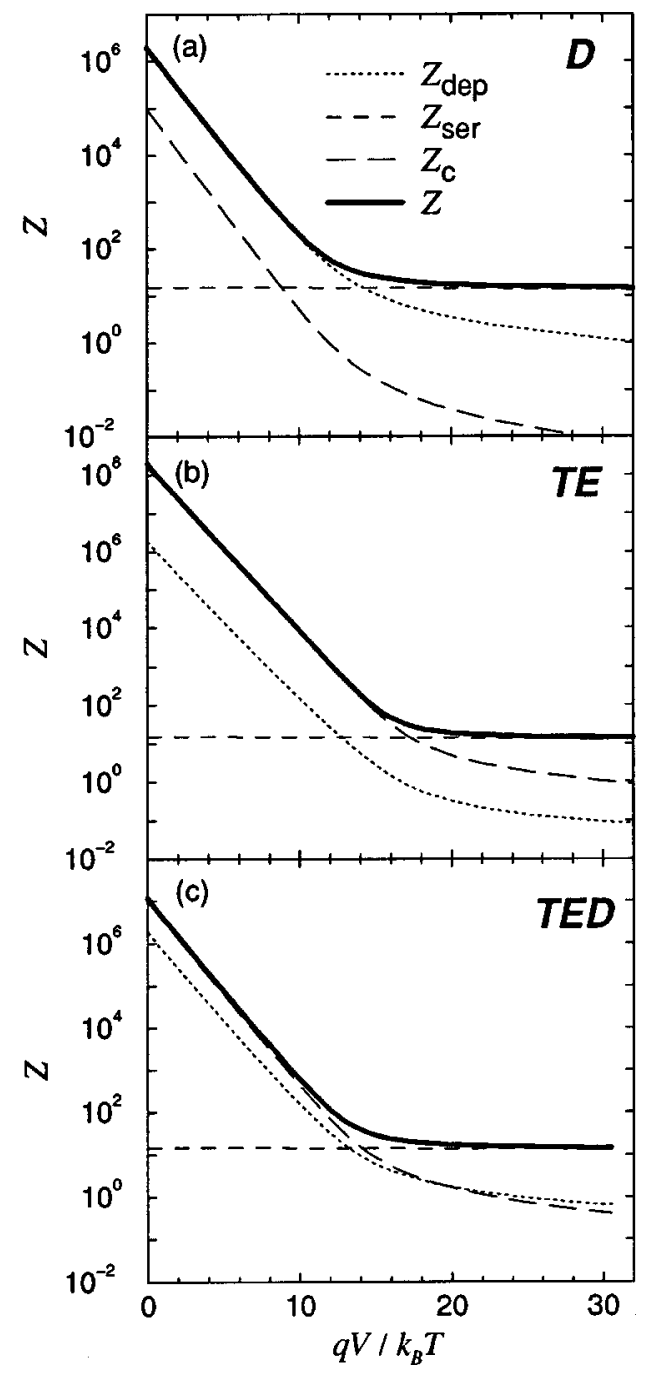

FIG. 7. Impedance $Z$ vs applied voltage $V$ at different regimes: D (a), TE-D (b), and TE (c). The relative contributions from the contact $Z_{c}$ and the bulk $Z_{\text {ser }}, Z_{\text {dep }}$ are compared.

values of the current. The term $\nabla Z_{\text {dep }}(x)$ is seen to be mostly localized in the depletion layer and it plays the main role at low currents whenever the depletion layer is appreciable. On the other hand, the spatial profile for $\nabla Z_{\text {ser }}(x)$ is flat over almost the whole structure with the magnitude corresponding to the donor density. It is seen that its shape depends weakly on the bias. This clearly explains why this term represents the series resistance of the sample.

Integrating along the structure the impedance-field components, the depletion impedance $Z_{\mathrm{dep}}$, and the series impedance $Z_{\text {ser }}$ are computed. By adding them to the contact impedance $Z_{c}$, the total impedance $Z$ is evaluated. Figure 7 shows the results as functions of the applied bias in the different transport regimes. It is seen, that for low biases the main contribution comes from either $Z_{\text {dep }}$ (case D) or $Z_{c}$ (case TE) (or both at TE-D regime), while at high voltages the series impedance $Z_{\text {ser }}$ always dominates. Note that the series impedance $Z_{\text {ser }}$ is almost constant in the whole range of voltages considered.

A similar analysis is performed for the voltage fluctuations. As before, the meaning of the different terms can be 


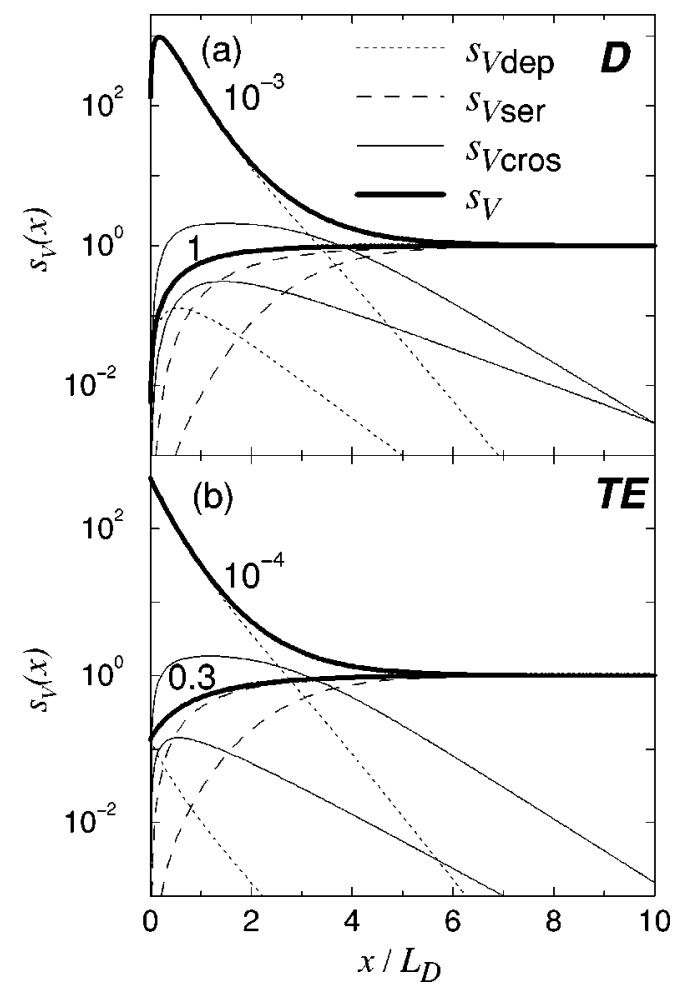

FIG. 8. Spatial profiles for the local noise $s_{V}(x)$ [normalized by $\left.4 k_{B} T \Delta f /\left(\mu A N_{D}\right)\right]$ in regimes D (a) and TE (b). For each type of line, the curve on top corresponds to the lower current.

justified by means of the local noise analysis of the diode. In Figs. 8(a) and 8(b) the local noise distributions $s_{V}(x)=s_{V \text { dep }}(x)+s_{V \text { cros }}(x)+s_{V \text { ser }}(x)$ are depicted for two different transport regimes (D and TE) and for two different values of the current. It is seen that the term $s_{V \text { dep }}$ is mainly localized in the depletion region, the term $s_{V \text { ser }}$ dominates in the quasineutral part of the sample, and $s_{V \text { cros }}$ is important in the crossover between the depletion and the quasineutral regions and represents their cross-correlation. At low currents the depletion contribution dominates, while at high currents, when the depletion layer has disappeared, all the components are comparable in magnitude. The main difference between the $\mathrm{D}$ and TE cases is in the position of the local noise maxima at low currents. For the case TE it lies at the interface $x=0$ where the electron density is lowest, while for the case $\mathrm{D}$ it is displaced towards the depletion region, since for this case the carrier density at $x=0$ is fixed by the boundary condition and does not fluctuate significantly.

Integrating along the device the spatial profiles for all the components, the spectral densities of voltage fluctuations $S_{V \text { dep }}, S_{V \text { cros }}$, and $S_{V \text { ser }}$ are found. To get the total voltage noise they should be added to the contact noise term $S_{V \mathrm{c}}$, which is localized at the metal-semiconductor interface. The relative significance of different components for different applied biases is shown in Fig. 9. For low voltages the main contribution comes from either $S_{V \text { dep }}$ (case D) or $S_{V \mathrm{c}}$ (case TE) (or $S_{V \mathrm{c}}+S_{V \text { dep }}$ for TE-D case), while at high voltages $S_{V \text { ser }}$ dominates. At intermediate biases $S_{V \text { cros }}$ is seen to contribute appreciably.

Having found the total impedance $Z$ and the voltagenoise spectral density $S_{V}$, the spectral density of current

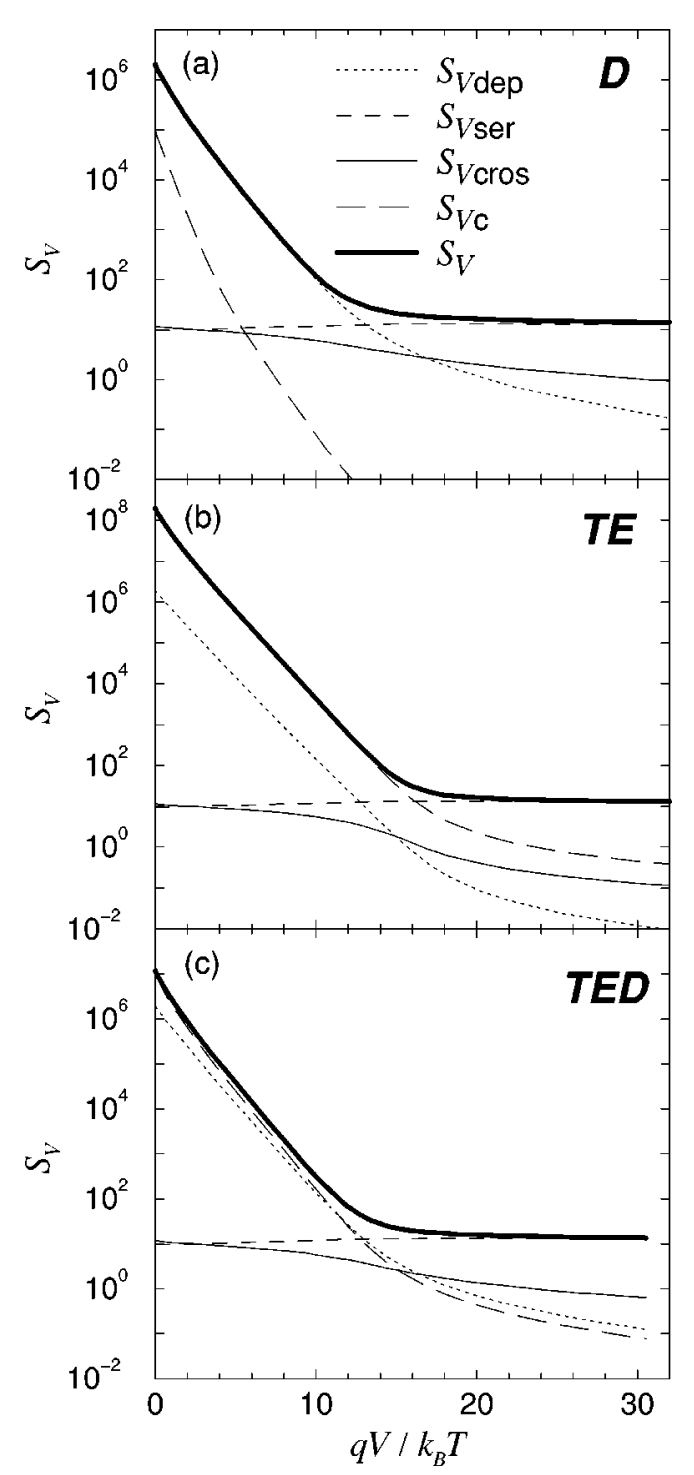

FIG. 9. Voltage fluctuations $S_{V}$ vs applied voltage $V$ at different regimes: D (a), TE-D (b), and TE (c). The relative contributions from the contact $S_{V \mathrm{c}}$ and the bulk $S_{V \text { ser }}, S_{V \text { dep }}, S_{V \text { cros }}$ are compared (all normalized to $4 k_{B} T R_{R}$ ).

fluctuations $S_{I}$ is computed from Eq. (29). The results for different transport regimes are shown in Fig. 10. A first important point to be noted is that in all cases the current fluctuations display a similar behavior: for very low current levels $\left(J \leq I_{c} / I_{R}=\alpha / \beta\right) S_{I}$ is constant, since it gives the thermal equilibrium noise with different diode resistances according to different $\beta$. Whereas at higher currents $\left(\alpha / \beta \leqq J \lesssim 10^{-2}\right)$ all the curves exhibit a $2 q I$ shot-noise-like behavior. ${ }^{19}$

These two different behaviors can in fact be predicted explicitly from our analytical results. Indeed, by neglecting at low biases $\left(V \leqq V_{\mathrm{bi}}\right)$ the series-resistance and cross contributions, one gets

$$
S_{I} \approx \frac{S_{V \mathrm{c}}+S_{V \mathrm{dep}}}{\left(Z_{c}+Z_{\mathrm{dep}}\right)^{2}},
$$

which in the depletion approximation, valid under this lowvoltage regime, yields (see Appendix B)

$$
S_{I}=2 q\left[I+2 I_{\mathrm{sat}}\right]
$$




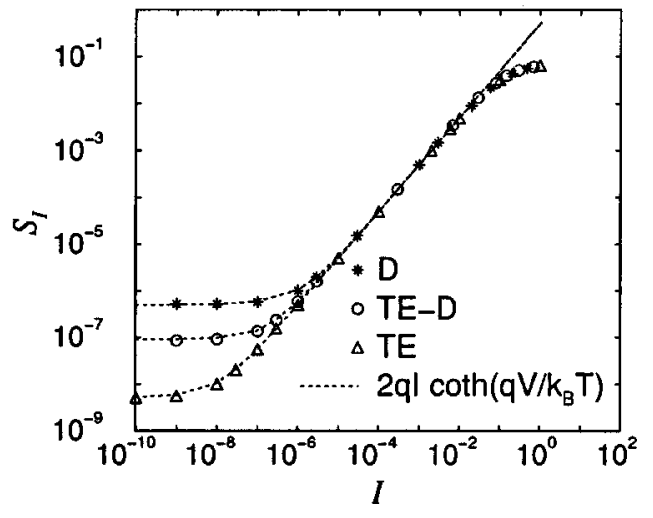

FIG. 10. Current-noise spectral density $S_{I}$ vs current $I$ at different regimes: $\mathrm{D}, \mathrm{TE}-\mathrm{D}$, and TE. The low-current limit is shown to be nicely described by Eq. (45) (dashed line). $S_{I}$ and $I$ are normalized by $4 q I_{R}$ and $I_{R}=q \mu E_{t h} N_{D} A$, respectively.

$$
\approx 2 q I, \quad \text { for } I \gg I_{\text {sat }},
$$

where $I_{\text {sat }}$ is the saturation current of the diode which includes both the effects of thermionic-emission and diffusion processes, and it is given by Eq. (B13). Note, that in general, $I_{\text {sat }}$ is a voltage dependent function due to the diffusion effects. It is worth noting that the validity of Eq. (42) extends over not only the ohmic regime but the hot-electron regime as well (see Appendix B). For the case of ohmic transport, however, the $I-V$ characteristics takes on a simple form ${ }^{4}$

$$
I=I_{\text {sat }}\left[\exp \left(q V / k_{B} T\right)-1\right],
$$

and Eq. (42) can be rewritten as ${ }^{9}$

$$
S_{I}=2 q I \operatorname{coth}\left(q V / 2 k_{B} T\right),
$$

which does not include explicitly any system dependent parameter. It can be seen that the data plotted in Fig. 10 closely follow this law independently on the transport regime considered.

At high biases ( $\left.V>V_{\mathrm{bi}}\right)$ the current fluctuations $S_{I}$ deviate from the $2 q I$ law and tend to saturate. In this limit we may approximate $Z \approx Z_{\text {ser }}$ and $S_{V} \approx S_{V \text { ser }}=4 k_{B} T Z_{\text {ser }}$, which are independent on the bias (see Figs. 7 and 9). Consequently, $S_{I}=4 k_{B} T / Z_{\text {ser }}$ is independent of the current, that explains the saturation behavior at high voltages.

The effective noise temperature $T_{n}$ as a function of bias in different transport regimes is depicted in Fig. 11. One can see, that starting from $T_{n}=T$ at zero bias (thermal equilibrium noise) it drops sharply towards $T / 2$ for low voltages that is a consequence of the $2 q I$ law and is typical for the shot noise behavior. ${ }^{5,9}$ At higher biases $T_{n}$ increases again approaching the ambient temperature $T$, corresponding to the diffusion noise of the series resistance. Note that $T_{n}$ may attain $T$ or may not, depending on the length of the Schottky diode $L$ (the magnitude of the series resistance). ${ }^{14}$ The noise temperature $T_{n}$ can be used as a figure of merit in order to evaluate the effects of the different sources of noise on the device performance. In particular, it can be seen that the more the transport is thermionic-emission dominated, the more $T_{n}$ approaches the value $T / 2$. In this sense, one can conclude that the TE case is less noisy than the D case.

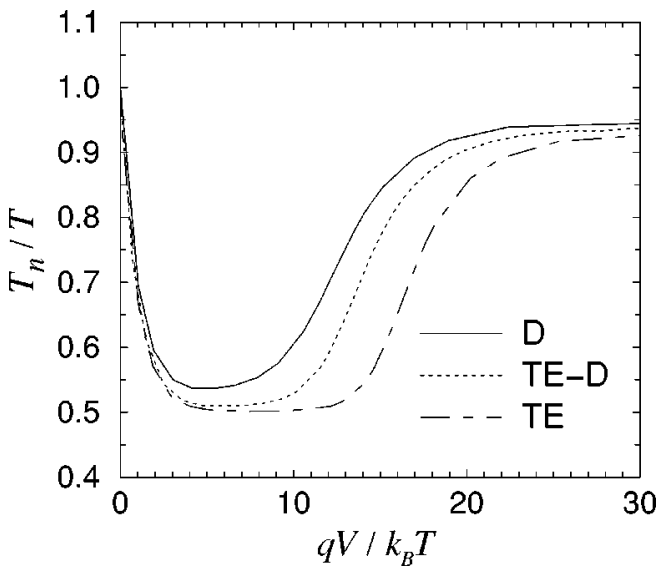

FIG. 11. Noise temperature $T_{n}$ vs applied voltage $V$ at different regimes: D, TE-D, and TE.

The importance of the cross-correlation term can be made more explicit by plotting $S_{I}$ and $T_{n}$ with and without including this term. Figure 12 clearly shows that both functions deviate significantly starting from the voltage $V \approx 10 k_{B} T / q\left(J \approx 10^{-2}\right)$ when the series effects starts to appear. A similar behavior has been observed in some experiments, ${ }^{6}$ where the noise temperature was found to deviate from the equivalent circuit results (without the cross term) precisely as shown in Fig. 12. Therefore, a part of the excess noise observed could be assigned to the crosscorrelations between the different noise sources. This statement is further confirmed by the recent MC simulation, ${ }^{8}$ which also found out the discrepancies with the equivalent circuit results (compare Fig. 12 of the present article with Fig. 3 of Ref. 8).

\section{DISCUSSION}

In this article we have presented an analytical analysis of the noise properties of an $n$-type Schottky barrier diode under forward bias conditions. Our study has been based upon a noise model which incorporates in a unified way both the noise due to the diffusion of carriers through the semiconductor and the noise due to the thermionic-emission of car-

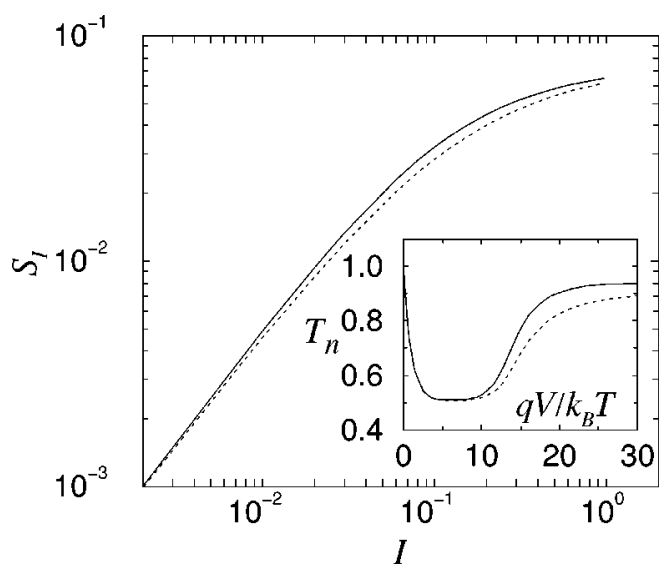

FIG. 12. Current-noise spectral density $S_{I}$ vs current $I$ for the regime TE-D with (solid line) and without (dashed line) the cross-correlation term. Inset: The same for the noise temperature $T_{n}$. 
riers across the metal-semiconductor interface. By means of the analytical solution of the model, the different contributions to the net noise of the diode have been identified, and with the help of a local analysis, assigned to different spatial regions of the device. Furthermore, the relevance of the different contributions depending on the characteristic parameters and the applied voltage range has been analyzed. In particular, it has been shown that at low biases $\left(V \lesssim V_{\mathrm{bi}}\right)$ the noise properties are dominated either by the diffusion of carriers in the depletion layer near the interface $(\beta \ll 1)$ or by the thermionic emission of carriers across the metalsemiconductor interface $(\beta \gg 1)$. Here, $\beta$ is the coefficient between the diffusion velocity and the contact recombination velocity. At high biases $\left(V \geqslant V_{\mathrm{bi}}\right)$, the noise properties are shown to be dominated in all cases by the diffusion of carriers through the quasineutral region of the device. Finally, starting from the applied voltage $V \sim V_{\mathrm{bi}}$ at which the depletion layer becomes small, an additional contribution has been identified. Due to its spatial localization in the crossover between the depletion layer and quasineutral region of the device, this additional term has been argued to represent the cross-correlation between the voltage noise sources originated in these two parts of the device.

A detailed comparison between the results predicted by means of our model and those predicted by means of the conventional equivalent circuit noise technique has been performed. In particular it has been shown that:

(i) The so-called junction resistance $R_{j}$, whose origin is not clearly established in circuit analysis, consists in general of two contributions: $Z_{c}$ and $Z_{\text {dep }}$, for which we give explicit expressions.

(ii) The series resistance $R_{s}$ is given through $Z_{\text {ser }}$, whose value can also be obtained from the corresponding expression.

(iii) The $2 q I R_{j}^{2}$ behavior associated with $R_{j}$ at low current levels is in fact given through $S_{V \mathrm{c}}+S_{V \text { dep }}$, which reproduces this type of behavior at low voltages, but holds in the whole range of biases. Note, that both the thermionic emission of carriers through the metal-semiconductor interface as well as the diffusion of carriers in the depletion region contribute to this behavior.

(iv) The noise due to the series resistance is given through $S_{V \text { ser }}$, which reproduces correctly the well known thermal noise behavior.

(v) There is an additional contribution $S_{V \text { cros }}$ to the voltage fluctuations not present in the conventional equivalent circuit analysis. This contribution is due to the cross-correlation between the fluctuations generated in the depletion layer and those in the quasi-neutral region of the semiconductor. It starts to become relevant in the transition from the $2 q I$ behavior towards the series-resistance noise (near the flat-band conditions). It has been argued that this additional term could explain some discrepancies observed between experimental measurements or MC simulations and the results of the equivalent circuit model precisely under near flat-band conditions. It should be pointed out that mm-wave and sub-mmwave mixers operate precisely at this regime, ${ }^{7}$ so that our theory can be useful for the local-noise analysis of these devices in order to improve their performance.
It is worth remarking that our theory provides closed analytical expressions to evaluate all the terms appearing in the impedance as well as in the voltage and current fluctuations under the full range of applied biases. Hence, it can be used to improve the existing equivalent circuit model. These expressions can in fact be easily inserted into the developed numerical programs which calculate the steady $I-V$ characteristics and electric field profiles for Schottky diodes, thus adding also information about the local and global noise properties of these systems. In addition, the method presented in this article, besides its fruitful application for the Schottky barrier diodes, it can be incorporated into any device model described by means of the drift-diffusion approach and its modifications. ${ }^{21}$ Our work then offers new perspectives on what concerns the spatial analysis of the noise properties of space-charge-limited devices, such as $n^{+}-n-n^{+}$diodes, field-effect transistors, etc.

\section{ACKNOWLEDGMENTS}

Two authors (O.M.B. and G.G.) acknowledge support by the Generalitat de Catalunya. O.M.B. is also grateful to Dirección General de Enseñanza Superior of Spain for financial support. This was was supported by the DGICYT of the Spanish Government under Grant No. PB95-0881.

\section{APPENDIX A: SPECTRAL DENSITY OF LOW- FREQUENCY CURRENT FLUCTUATIONS AT A METAL-SEMICONDUCTOR INTERFACE}

The current crossing at the metal-semiconductor interface consists of electrons emitted individually and at random in both directions. If $I_{\mathrm{ms}}=q n_{0}^{\mathrm{eq}} v_{r} A$ is the current from metal to semiconductor and $I_{\mathrm{sm}}=q n_{0} v_{r} A$ is the current from semiconductor to metal, then the net current is $I=I_{\mathrm{sm}}-I_{\mathrm{ms}}$ as given by formula (3). Since both random processes are assumed to be statistically independent $I_{\mathrm{ms}}$ and $I_{\mathrm{sm}}$ exhibit full shot noise. ${ }^{9}$ Therefore, for the low-frequency spectral density of current fluctuations generated at the metal-semiconductor interface one gets

$$
S_{I \mathrm{c}}=2 q\left(I_{\mathrm{sm}}+I_{\mathrm{ms}}\right)=2 q\left(I+2 I_{\mathrm{ms}}\right)=2 q\left(I+2 I_{c}\right),
$$

where $I_{c} \equiv I_{\mathrm{ms}}$.

\section{APPENDIX B: UNIVERSALITY OF THE $2 q I$ LAW FOR THE CURRENT NOISE IN DIFFERENT TRANSPORT REGIMES}

The purpose of this appendix is to show explicitly from our analytical results that the $2 q I$ behavior in the current spectral density $S_{I}$ observed at low currents emerges not only in the thermionic-emission case, as it would be expected, but also for the diffusion case and the combined TE-D case as well.

Neglecting the series resistance effects at low currents we have

$$
S_{I} \approx \frac{S_{V \mathrm{c}}+S_{V \mathrm{dep}}}{\left(Z_{c}+Z_{\mathrm{dep}}\right)^{2}} .
$$


Furthermore, for these values of the applied bias the depletion-layer approximation holds for which the electron concentration inside the depletion layer is neglected, since $n(x) \ll N_{D} \cdot{ }^{2}$ For this case $\rho(x)=d E(x) / d x$ becomes

$$
\rho(x) \approx \begin{cases}\rho_{0}, & 0<x<w, \\ 0, & w<x<L,\end{cases}
$$

where $\rho_{0}=q N_{D} / \epsilon$ and $w$ is the length of the depletion layer. Under these conditions the electric field is approximated as ${ }^{2}$

$$
E(x) \approx\left\{\begin{array}{l}
E_{0}(1-x / w), \quad 0<x<w, \\
0, \quad w<x<L .
\end{array}\right.
$$

As a result for the depletion-layer impedance by using Eq. (21) we have

$$
Z_{\mathrm{dep}}=\frac{E_{0}-E_{L}}{\epsilon A \rho_{0}^{\prime}} \int_{0}^{w} \frac{d x}{D(x) W(x)} \equiv \frac{C_{E}}{v_{D}},
$$

where $C_{E}=\left(E_{0}-E_{L}\right) /\left(\epsilon A \rho_{0}^{\prime}\right)$, and we have defined the diffusion velocity $v_{D}$ by ${ }^{22}$

$$
\frac{1}{v_{D}}=\int_{0}^{w} \frac{d x}{D(x) W(x)} .
$$

The expressions for the contact impedance and voltage spectral density follows from Eqs. (19) and (25),

$$
\begin{aligned}
& Z_{c}=\frac{C_{E}}{v_{r}}, \\
& S_{V \mathrm{c}}=C_{E}^{2} \frac{2 q\left[I+2 I_{c}\right]}{v_{r}^{2}}=C_{E}^{2}\left[\frac{2 q I}{v_{r}^{2}}+\frac{4 q^{2} A n_{0}^{\mathrm{eq}}}{v_{r}}\right] .
\end{aligned}
$$

Now, the only unknown term in Eq. (B1) is the depletion voltage-noise spectral density $S_{V \text { dep }}$ which can be found from Eq. (26)

$$
S_{V \mathrm{dep}}=4 q^{2} C_{E}^{2} A \int_{0}^{w} \frac{n(x) d x}{D(x) W^{2}(x)} .
$$

To proceed further we integrate Eq. (1) with the help of the integrating factor $1 / W(x)$ finding the following relation:

$$
\frac{n(x)}{W(x)}=n(0)+\frac{I}{q A} \int_{0}^{x} \frac{d x^{\prime}}{D\left(x^{\prime}\right) W\left(x^{\prime}\right)} .
$$

Substituting this relation into Eq. (B8) and using the identity (can be proved by integrating by parts)

$$
\int_{0}^{w} f(x)\left[\int_{0}^{x} f\left(x^{\prime}\right) d x^{\prime}\right] d x=\frac{1}{2}\left[\int_{0}^{w} f(x) d x\right]^{2},
$$

with $f(x)=1 /[D(x) W(x)]$, one arrives at

$$
S_{V \text { dep }}=4 q^{2} C_{E}^{2} A\left(\frac{n(0)}{v_{D}}+\frac{I}{2 q A v_{D}^{2}}\right) .
$$

Now we can use the boundary condition for $n(0)$ given by Eq. (3) and substitute all the terms into Eq. (B1). One gets

$$
S_{I}=2 q\left[I+2 \frac{q A n_{0}^{\mathrm{eq}} v_{r} v_{D}}{v_{r}+v_{D}}\right] \equiv 2 q\left[I+2 I_{\text {sat }}\right],
$$

where

$$
I_{\text {sat }}=q A n_{0}^{\mathrm{eq}} \frac{v_{r} v_{D}}{v_{r}+v_{D}}
$$

is the saturation current of the diode including both the effects of thermionic-emission and diffusion processes. Note the universality of Eq. (B12) which holds for different transport regimes (D, TE, and TE-D) and conduction mechanisms (ohmic, hot-electrons). For the D case under ohmic conditions a similar derivation of the formula (B12) has been performed by van der Ziel. ${ }^{23}$

${ }^{1}$ T. W. Crowe, R. J. Mattauch, H. P. Röser, W. L. Bishop, W. C. B. Peatmean, and X. Liu, Proc. IEEE 80, 1827 (1992).

${ }^{2}$ E. H. Rhoderick and R. H. Williams, Metal-Semiconductor Contacts, 2nd ed. (Clarendon Press, Oxford, 1988).

${ }^{3}$ H. K. Henisch, Semiconductor Contacts (Clarendon Press, Oxford, 1984).

${ }^{4}$ S. M. Sze, Physics of Semiconductor Devices, 2nd ed. (Wiley, New York, 1981).

${ }^{5}$ M. Trippe, G. Bosman, and A. van der Ziel, IEEE Trans. Microwave Theory Tech. 34, 1183 (1986).

${ }^{6}$ A. Jelenski, E. L. Kollberg, and H. H. G. Zirath, IEEE Trans. Microwave Theory Tech. 34, 1193 (1986).

${ }^{7}$ A. Jelenski, A. Grüb, V. Krozer, and H. L. Hartnagel, IEEE Trans. Microwave Theory Tech. 41, 549 (1993).

${ }^{8}$ T. González, D. Pardo, L. Varani, and L. Reggiani, Appl. Phys. Lett. 63, 3040 (1993); J. Appl. Phys. 82, 2349 (1997).

${ }^{9}$ A. van der Ziel, Noise in Solid State Devices and Circuits (Wiley, New York, 1986)

${ }^{10}$ O. M. Bulashenko, G. Gomila, J. M. Rubí, and V. A. Kochelap, Appl. Phys. Lett. 70, 3248 (1997); J. Appl. Phys. 83, 2610 (1998).

${ }^{11}$ M. J. Martín, T. González, D. Pardo, and J. E. Velázquez, Semicond. Sci. Technol. 11, 380 (1996).

${ }^{12}$ C. R. Crowell and S. M. Sze, Solid-State Electron. 9, 1035 (1966); Reprinted in Semiconductor Devices: Pioneering Papers, edited by S. M. Sze (World Scientific, Singapore, 1991), pp. 414-427.

${ }^{13}$ An extension of this boundary condition to the case of non-ideal metalsemiconductor contacts has been worked out in G. Gomila, A. PérezMadrid, and J. M. Rubí, Physica A 233, 208 (1996).

${ }^{14}$ G. Gomila, O. M. Bulashenko, and J. M. Rubí (unpublished).

${ }^{15}$ This reasoning may be made more rigorous by considering the nonequilibrium thermodynamics arguments. For a general formulation of the theory, see e.g.: S. R. de Groot and P. Mazur, Non-equilibrium Thermodynamics (Dover, New York, 1984). For its application to semiconductor systems, see G. Gomila and J. M. Rubí, Physica A 234, 851 (1997).

${ }^{16}$ C. M. van Vliet, IEEE Trans. Electron Devices 41, 1902 (1994).

${ }^{17}$ K. M. van Vliet, J. Math. Phys. 12, 1981 (1971).

${ }^{18}$ K. M. van Vliet and H. Mehta, Phys. Status Solidi B 106, 11 (1981).

${ }^{19}$ It is the custom to call each type of noise with the current spectral density $S_{I}=2 q I$ as a shot noise. Actually, the genuine shot noise is related to the Poissonian ballistic electron flow like that occurred in vacuum-tube diodes (Ref. 9), or in ballistic semiconductor devices (Ref. 20). Here, this relation appears as a consequence of the (near-)exponential $I-V$ characteristics under combined diffusive and thermionic-emission transport regimes, which are apparently not ballistic. To use the term "shot" to characterize the type of noise under this situation is not completely adequate.

${ }^{20}$ T. González, O. M. Bulashenko, J. Mateos, D. Pardo, and L. Reggiani, Phys. Rev. B 56, 6424 (1997).

${ }^{21}$ P. J. Price, J. Appl. Phys. 63, 4718 (1988).

${ }^{22}$ For ohmic conduction our definition reduces to the conventional one (Ref. 4): $v_{D}=\left(\mu k_{B} T / q\right)\left\{\int_{0}^{w} \exp \left[q \phi(x) / k_{B} T\right] d x\right\}^{-1}$ where $\phi(0)=0$ has been used.

${ }^{23}$ A. van der Ziel, Physica B 94, 357 (1978). 